\title{
Tissue and Cellular Basis for Impaired Bone Formation in Aluminum-related Osteomalacia in the Pig
}

\author{
Aileen B. Sedman, Allen C. Alfrey, Nancy L. Miller, and William G. Goodman \\ Department of Pediatrics, University of Michigan Medical School, Ann Arbor, Michigan 48109; \\ Medical and Research Services, Veterans Administration Medical Center, and University of Colorado School of Medicine, \\ Denver, Colorado 80220; Medical and Research Services, Sepulveda Veterans Administration Medical University Center, \\ School of Medicine/San Fernando Valley Program, Sepulveda, California 91343
}

\begin{abstract}
Bone formation is impaired in aluminum-associated bone disease. Reductions in the number of osteoblasts or in the function of individual osteoblasts could account for this finding. Thus, quantitative bone histology and measurements of bone formation were done at three skeletal sites in piglets given aluminum (Al) parenterally, $1.5 \mathrm{mg} / \mathrm{kg}$ per d, for 8 wk $(\mathrm{Al}, n=4)$ and in control animals $(C, n=4)$. Bone $A l$ was $241 \pm 40 \mathrm{mg} / \mathrm{kg}$ per dry weight in $\mathrm{Al}$ and 1.6 \pm 0.9 in $\mathrm{C}, P<0.001$. All Al-treated animals developed osteomalacia with increases in osteoid seam width, osteoid volume, and mineralization lag time at each skeletal site, $P<0.05$ vs. $C$ for all values. Mineralized bone formation at the tissue level was lower in Al than in $\mathbf{C}, P<0.05$ for each skeletal site, due to reductions in active bone forming surface. Bone formation at the cellular level was similar in each group, however, and total osteoid production by osteoblasts did not differ in $\mathbf{C}$ and Al. Aluminum impairs the formation of mineralized bone in vivo by decreasing the number of active osteoblasts, and this change can be distinguished from the effect of aluminum to inhibit, either directly or indirectly, the calcification of osteoid.
\end{abstract}

\section{Introduction}

Reductions in the rate of formation of bone are a hallmark of aluminum-related bone disease, but the pathogenesis of this disturbance remains uncertain (1-6). In aluminum-associated osteomalacia, bone formation is markedly reduced from normal values when evaluated by the technique of double tetracycline labeling (1-5). Histologic evidence of osteoid accumulation and widened osteoid seams are also prominent features of this disorder $(1,3,5)$. In contrast, the volume of osteoid is normal or reduced in the aplastic lesion of aluminum-associated bone disease despite reductions in the rate of bone formation similar to those observed in osteomalacia $(3,5,6)$. Since bone formation may be suppressed in aluminum-related bone disease without concurrent histologic evidence of osteoid accumulation, it is possible that aluminum adversely affects the formation of bone and/or the synthesis of collagen in a manner independent of the putative role of aluminum to inhibit the process of mineralization.

Bone formation at the tissue level may be impaired as a result of reductions in the cellular activity of individual osteo-

Address reprints requests to Dr. Goodman, Nephrology Section (111R), Sepulveda Veterans Administration Medical Center, 16111 Plummer Street, Sepulveda, CA 91343. 1986

Received for publication 8 October 1985 and in revised form 11 July

The Journal of Clinical Investigation, Inc.

Volume 79, January 1987, 86-92 blasts or as a consequence of reductions in the total number of active osteoblasts $(7,8)$. Observations from clinical bone biopsy material indicate that the extent of active bone-forming surface is reduced in both the osteomalacic and aplastic lesions of aluminum-associated bone disease as judged by diminished or absent tetracycline uptake into bone $(3,5,6,9)$. Such findings suggest that the number of osteoblasts is reduced in these two disorders. However, several investigators have reported that the rate of apposition of mineralized bone is diminished in aluminum-related bone disease $(2,4,6,10,11)$. These data are consistent with the view that the cellular activity of individual osteoblasts is adversely affected by the accumulation of aluminum in bone.

Previous studies of bone formation and bone histology in experimental animals given aluminum for varying periods have been hampered by the virtual absence of tetracycline uptake into bone at sites of skeletal remodeling (12-14). Precise measurements of the rates of bone formation and apposition during aluminum loading thus are limited, particularly for trabecular, or cancellous bone where the histologic changes of aluminumrelated bone disease are most prominent (15). In the current investigation, growing piglets were given repeated intravenous injections of aluminum for $8 \mathrm{wk}$. Despite the development of overt histologic osteomalacia, double tetracycline labeling of bone was achieved, and measurements of the rates of bone formation and apposition were possible in this experimental model. The results indicate that the formation of bone at the cellular level is not impaired during aluminum loading; however, the number of active bone forming sites within skeletal tissue is diminished. This disturbance is primarily responsible for the low rate of mineralized bone formation in aluminum-related bone disease.

\section{Methods}

Experimental protocol. Eight littermate pigs (Yorkshire strain, Amo Farms, Ann Arbor, MI) were obtained at 5 wk of age and housed in individual pens for the duration of study. All animals had free access to water and were maintained on standard pig chow (Pig Growena, Ralston Purina Co., Chicago, IL) containing $1.6 \%$ calcium and $0.57 \%$ phosphorus. Prior to study, indwelling polyethylene catheters were surgically placed in the left jugular vein of all animals while under anesthesia with ketamine and halothane. The catheters coursed through the subcutaneous tissue and exited externally from the skin at a site above the left shoulder. Patency of the venous catheters was maintained throughout the study by daily irrigations with heparinized saline.

Individual pigs were matched by weight and assigned to one of two study groups, control $\left(\mathrm{C}^{1} n=4\right)$ and aluminum-treated $(\mathrm{Al}, n=4) .1$

1. Abbreviations used in this paper: $\mathrm{C}$, control. 
wk after the placement of intravenous catheters, injections of aluminum were begun. Aluminum chloride was added to deionized water as the vehicle, and the solution was administered to animals in Al by slow intravenous injection over a 10 -min period $7 \mathrm{~d}$ each week for $8 \mathrm{wk}$. The daily dose of aluminum was adjusted to provide $1.5 \mathrm{mg}$ of elemental aluminum $/ \mathrm{kg}$ body per $\mathrm{d}$. Control animals received daily infusions of vehicle only. To assure similar degrees of weight gain between groups, animals in $\mathrm{C}$ were pair-fed with those in $\mathrm{Al}$.

All animals were given single intravenous injections of tetracycline $\mathrm{HCl}, 20 \mathrm{mg} / \mathrm{kg}, 8 \mathrm{~d}$ and $1 \mathrm{~d}$, respectively, before killing at the conclusion of the study $(10,11,14) .2 \mathrm{~d}$ before termination of the animals, 24-h collections of urine were obtained, the volume was measured, and aliquots were saved for subsequent biochemical determinations. The animals were killed by exsanguination by cardiac puncture while under anesthesia using ketamine and pentobarbital. Samples of serum were saved for biochemical determinations.

Histologic techniques. Recent observations from this laboratory indicate that the histologic findings in bone may differ at various sites within the skeleton following the administration of aluminum to experimental animals (15). In the current study, therefore, quantitative histology was done on specimens of trabecular bone obtained from three different skeletal sites, two in the proximal tibia and one in the distal femur. Each site was selected on the basis of preliminary studies in normal pigs similar in age to the animals used in the current investigation. These studies indicated that the areas within the skeleton chosen for histologic evaluation were comprised exclusively of trabecular bone.

Immediately after the animals were killed at the conclusion of the study, bone biopsies were obtained from the metaphysis of the distal femur using a Bordier trephine. The site of biopsy was standardized to an area on the lateral surface of the left femur $4 \mathrm{~cm}$ proximal to the lateral femoral condyle; the biopsy was taken with the trephine oriented perpendicular to longitudinal axis of the bone and directed medially. Bone biopsy specimens were fixed for $24 \mathrm{~h}$ in $10 \%$ phosphate-buffered formalin, dehydrated in ethanol, infiltrated with glycol-methylmethacrylate, and polymerized at $4^{\circ} \mathrm{C}$ as described previously from this laboratory $(14,15)$.

The left tibia was also removed intact from all animals, stripped of soft tissue, wrapped with gauze saturated with $10 \%$ formalin, and stored at $4^{\circ} \mathrm{C}$ until blocks of tissue were prepared for embedding. Bone for histologic evaluation was obtained from both the epiphysis of the proximal tibia, above the growth plate, and the metaphysis of the proximal tibia, $\sim 4 \mathrm{~cm}$ beneath the growth plate. Cuboidal specimens of trabecular bone measuring 1.0-1.5 $\mathrm{cm}$ were prepared from each site using a circular saw (Beuhler Isomet, Beuhler Ltd., Evanston, IL). These were placed in $70 \%$ ethanol, dehydrated, and processed for tissue embedding as outlined above for specimens of femoral bone $(14,15)$.

Sections of nondecalcified bone were prepared from plastic embedded tissues using a sledge microtome (June Autocut 1140, Reichert-Jung, Vienna, Austria) $(14,15)$. The plane of section for all specimens was parallel to the longitudinal axis of the bone. $5-\mu \mathrm{m}$ sections of bone were stained using the modified Goldner technique and examined by light microscopy (14-16); 10- $\mu \mathrm{m}$ sections were mounted unstained in $10 \%$ glycerol and viewed by epifluorescent illumination for the evaluation of tetracycline labels $(14,15) .5-\mu \mathrm{m}$ sections were also stained by the aurine tricarboxylic acid method for histochemical determinations of the aluminum content of bone $(9,14,15)$.

Bone measurements. Quantitative histology of bone was done using a digitizer (Summagraphics Corp., Fairfield, CT) interfaced with a microcomputer (IBM PC, IBM Instruments, IBM Corp., Danbury, CT) and a series of measuring programs $(10,11,14,15)$. All measurements of length, width, and area for trabecular bone were determined directly from images projected to the surface of the digitizer tablet using a projection prism and drawing tube attached to a microscope (Dialuz, Leitz, Wetzlar, Federal Republic of Germany) $(10,11,14,15)$.

The following measurements were obtained for trabecular bone from each skeletal site: $(a)$ total bone area (percent), the area of bone, including both mineralized bone and osteoid, expressed as a percentage of the total tissue area; $(b)$ osteoid area (percent), the area of osteoid expressed as a percentage of the total bone area; (c) osteoid seam width (micrometer), the mean width of osteoid seams and determined by dividing the total osteoid area, in square millimeters, by the length, in millimeters, of osteoid surface; $(d)$ osteoid surface (percent), the percentage of trabecular bone surfaces covered by osteoid; $(e)$ resorption surface (percent), the percentage of trabecular bone surfaces characterized by the presence of scalloped bone resorptive lacunae; $(f)$ double tetracycline labeled surface (percent), the percentage of trabecular bone surfaces that exhibited double bands of tetracycline fluorescence; $(g)$ single tetracycline labeled surface (percent), the percentage of bone surfaces that exhibited single bands of tetracycline fluorescence; and $(h)$ osteoblastic osteoid surface (percent), the percentage of osteoid surfaces characterized by the presence of a layer of large cuboidal cells at the surface of the osteoid seam. The bone aluminum content, determined by histochemical methods, was expressed as a percentage of the total bone surface staining positive for aluminum $(14,15)$.

Additional 5- $\mu \mathrm{m}$ sections of bone from the epiphysis were stained with toluidine blue (17) and examined by concurrent light and epifluorescence microscopy. The distance of the osteoid surface from the cement line, in micrometers, was determined at a minimum of 20 separate osteoid seams in tissue sections from each animal. Measurements were done both at osteoid surfaces adjacent to double tetracycline labels and at osteoid surfaces adjacent to unlabeled bone. The width of osteoid seams was also measured along each type of osteoid surface.

The rate of mineralized bone apposition was determined by dividing the width of separation of double tetracycline labels by the time interval between the administration of the two labels; the results are expressed in micrometers per day $(14,18)$. The width of separation of tetracycline labels was not corrected for obliquity of the plane of section (18). The rates of bone formation were calculated according to methods published previously $(7,8,18)$. Bone formation at the tissue level was expressed as the area of bone formed per unit area of existing trabecular bone, in square millimeter per square millimeter per day, and per unit length of osteoid surface, in square millimeter per millimeter per day; bone formation at the cellular level was expressed per unit length of active bone forming surface, in square millimeter per millimeter per day, as judged by the presence of double tetracycline labels adjacent to surface osteoid seams. Corrected rates of bone apposition were also determined using the formula: $\mathrm{MAR}_{\mathrm{c}}=\mathrm{MAR}_{\mathrm{u}} * \mathrm{Fract}_{1 \mathrm{lab}}$ where $\mathrm{MAR}_{\mathrm{c}}$ is the corrected rate of mineralized bone apposition, $\mathrm{MAR}_{\mathrm{u}}$ is the uncorrected rate of mineralized bone apposition as determined from the width of separation of double tetracycline labels, and Fract $_{1 \mathrm{ab}}$ is the fraction of osteoid surfaces labeled with tetracycline $(7,8)$. The total length of double tetracyclinelabeled bone surface plus one-half of the length of single tetracyclinelabeled surface was used for the calculation of $\operatorname{Fract}_{1 \mathrm{ab}}(7,18)$. The values

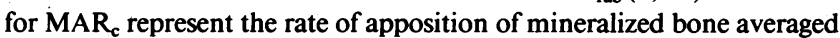
over the life span of an osteoid seam $(7,8)$. The mineralization lag time was calculated by dividing the mean osteoid seam width by the corrected rate of mineralized bone apposition; the results are expressed in days.

Biochemical measurements. The concentrations of calcium and phosphorus in serum were determined by automated methods (AutoAnalyzer, Beckman Instruments, Inc., Fullerton, CA) $(19,20)$. Serum and urine creatinine concentrations were measured using the modified Jaffe method (21). The aluminum content of bone was determined by flameless atomic absorption spectroscopy in specimens of trabecular bone from the metaphysis of the left femur (22). The levels of 1,25-dihydroxyvitamin $\mathrm{D}$ in serum were measured by methods described in detail elsewhere (23-25).

Statistical analysis. All results are expressed as the mean \pm 1 SD. Statistical analysis of the data was done using the $t$ test for unpaired samples and linear regression analysis (26). The Wilcoxon rank-sum test was used for comparisons between groups with unequal within-groups variances (26). One bone biopsy from the femoral metaphysis in Al contained only cortical and subcortical bone, and the values from this specimen were excluded from statistical analysis of the histologic and dynamic data for femoral bone. 
Table I. Serum Biochemistries and Weight Gains in C and Al Pigs*,

\begin{tabular}{lcc}
\hline & $\mathrm{C}$ & $\mathrm{Al}$ \\
\hline & $(n=4)$ & $(n=4)$ \\
Serum calcium, mg/dl & $9.2 \pm 0.3$ & $10.3 \pm 0.9^{8}$ \\
Serum phosphorus, $\mathrm{mg} / \mathrm{dl}$ & $7.7 \pm 0.5$ & $8.5 \pm 0.6^{8}$ \\
Serum creatinine, $\mathrm{mg} / \mathrm{dl}$ & $1.2 \pm 0.1$ & $1.2 \pm 0.1$ \\
Serum $1,25(\mathrm{OH}) 2 \mathrm{D}, \mathrm{pg} / \mathrm{ml}$ & $65.4 \pm 13.0$ & $76.6 \pm 42.3$ \\
Weight gain, $\mathrm{kg}$ & $8.8 \pm 5.9$ & $7.9 \pm 1.1$ \\
\hline
\end{tabular}

* Values are the mean \pm 1 SD.

${ }^{\ddagger}$ Probabilities determined by the $t$ test for unpaired samples.

$P<0.05$ vs. C.

\section{Results}

The serum concentrations of calcium and phosphorus increased from control values in animals given aluminum, but serum creatinine concentrations were similar in each group (Table I). The levels of 1,25-dihydroxyvitamin $\mathrm{D}$ in serum were also similar in $\mathrm{C}$ and $\mathrm{Al}$ (Table I). Serum immunoreactive parathyroid hormone (iPTH) levels were measured in all animals, but further validation of the results using various antisera to the PTH molecule in this species is required. Weight gains during the experiment did not differ between groups (Table I).

The aluminum content of bone, determined by chemical methods, was $1.6 \pm 0.9 \mathrm{mg} / \mathrm{kg}$ dry wt in C and $241 \pm 40 \mathrm{mg} / \mathrm{kg}$ dry wt in $\mathrm{Al}, P<0.001$. In control animals, the histochemical stain for aluminum was negative in all sections of bone examined. However, intense histochemical staining for aluminum was seen at $55.6 \pm 15.4 \%$ of trabecular bone surfaces in the femoral metaphysis, at $52.3 \pm 7.1 \%$ of bone surfaces in the tibial epiphysis, and at $57.2 \pm 12.5 \%$ of bone surfaces in the tibial metaphysis in $\mathrm{Al}$.
Osteomalacia was documented by histologic criteria in all aluminum-treated animals. At each skeletal site, the volume of osteoid was greater in $\mathrm{Al}$ than in $\mathrm{C}$ (Table II). The higher values for osteoid volume in Al were due both to increases in the width of osteoid seams and to increases in the extent of osteoid surface in trabecular bone (Table II). Consistent with the histologic findings of osteomalacia, the mineralization lag time was prolonged in $\mathrm{Al}$ when compared to control animals. In contrast to the increase in the values for percent osteoid surface in $\mathrm{Al}$, percent resorption surface at the epiphysis of the tibia and at the metaphysis of the femur was lower in $\mathrm{Al}$ than in $\mathrm{C}$ (Table II).

Double bands of tetracycline fluorescence were seen in sections of bone obtained from each skeletal site in all animals studied, but the extent of trabecular bone surface that exhibited double tetracycline labels was reduced from control values in Al (Table II). This finding was noted at each skeletal site. Thus, the number of sites of active bone formation was diminished in $\mathrm{Al}$ after $8 \mathrm{wk}$ of aluminum administration. Values for the percentage of osteoid surfaces covered with osteoblasts were also lower in $\mathrm{Al}$ than in $\mathrm{C}$. In the metaphysis of the femur, $37.7 \pm 4.1 \%$ of osteoid surfaces in control animals were characterized by the presence of osteoblasts whereas only $10.3 \pm 9.7 \%$ of osteoid surfaces exhibited osteoblasts in animals given aluminum, $P<0.01$. The values for percent osteoblastic osteoid correlated with the percentage of osteoid surfaces that exhibited double tetracycline labels $(r=0.92, P<0.01)$.

At all sites evaluated, the rate of mineralized bone formation was lower in $\mathrm{Al}$ than in $\mathrm{C}$ (Figs. 1 and 2). This was true whether the results were expressed per unit area of trabecular bone (Fig. 1) or per unit length of osteoid surface (Fig. 2). These findings indicate that mineralized bone formation at the tissue level was substantially reduced in animals given aluminum. The corrected rates of bone apposition were also lower in $\mathrm{Al}$ than in $\mathrm{C}$, and these data reflect the marked reduction in the extent of double tetracycline-labeled bone surface in $\mathrm{Al}$ (Fig. 3, Table II). However, the uncorrected rates of mineralized bone apposition, determined from measurements of the width of separation of dou-

Table II. Bone Histology and Tetracycline-based Bone Dynamics at Three Skeletal Sites in C and Al Pigs,*;

\begin{tabular}{|c|c|c|c|c|c|c|}
\hline & \multicolumn{2}{|c|}{ Femoral metaphysis } & \multicolumn{2}{|c|}{ Tibial epiphysis } & \multicolumn{2}{|c|}{ Tibial metaphysis } \\
\hline & $\begin{array}{l}C \\
(n=4)\end{array}$ & $\begin{array}{l}\text { Al } \\
(n=3)\end{array}$ & $\begin{array}{l}C \\
(n=4)\end{array}$ & $\begin{array}{l}\mathrm{Al} \\
(n=4)\end{array}$ & $\begin{array}{l}C \\
(n=4)\end{array}$ & $\begin{array}{l}\mathrm{Al} \\
(n=4)\end{array}$ \\
\hline Bone area, \% tissue area & $17.2 \pm 1.4$ & $21.7 \pm 4.1$ & $16.9 \pm 1.0$ & $18.8 \pm 2.1$ & $18.5 \pm 2.0$ & $21.3 \pm 2.8$ \\
\hline Osteoid area; $\%$ bone area & $2.7 \pm 0.7$ & $12.1 \pm 4.5^{8}$ & $2.8 \pm 0.9$ & $14.4 \pm 5.1^{8}$ & $3.1 \pm 1.5$ & $20.8 \pm 10.3^{8}$ \\
\hline Osteoid seam width, $\mu \mathrm{m}$ & $4.4 \pm 0.8$ & $10.0 \pm 4.8^{\prime \prime}$ & $4.2 \pm 1.0$ & $7.7 \pm 1.8^{8}$ & $3.6 \pm 1.1$ & $12.7 \pm 2.5^{* *}$ \\
\hline \multicolumn{7}{|l|}{ Osteoid surface, \% bone } \\
\hline surface & $21.2 \pm 6.4$ & $54.4 \pm 3.8^{\prime \prime}$ & $22.4 \pm 13.9$ & $61.0 \pm 12.9^{* *}$ & $27.3 \pm 8.3$ & $57.9 \pm 21.7^{8}$ \\
\hline $\begin{array}{l}\text { Resorption surface, \% bone } \\
\text { surface }\end{array}$ & $3.0 \pm 0.4$ & $1.3 \pm 0.6^{* *}$ & $3.5 \pm 1.4$ & $1.2 \pm 0.3^{8}$ & $6.7 \pm 2.5$ & $3.8 \pm 2.2$ \\
\hline \multicolumn{7}{|l|}{ Double tetracycline labeled } \\
\hline \multicolumn{7}{|l|}{ Single tetracycline labeled } \\
\hline surface, $\%$ bone surface & $7.5 \pm 2.0$ & $2.4 \pm 1.3^{6}$ & $3.6 \pm 1.0$ & $2.1 \pm 3.0$ & $4.5 \pm 2.6$ & $3.1 \pm 2.7$ \\
\hline Mineralization lag time, days & $3.6 \pm 0.8$ & $73.6 \pm 55.8^{\prime}$ & $1.5 \pm 0.2$ & $40.4 \pm 30.4^{\prime}$ & $1.7 \pm 0.9$ & $47.5 \pm 26.5^{9}$ \\
\hline
\end{tabular}

* Values are the mean \pm 1 SD. ${ }^{\ddagger}$ Probabilities determined by the $t$ test for unpaired samples except as indicated. ${ }^{8} P<0.05$ vs. C. $\quad{ }^{\top} P<0.05$ vs. $C$ by Wilcoxon rank-sum test. " $P<0.001$ vs. C. ** $P<0.01$ vs. C. 


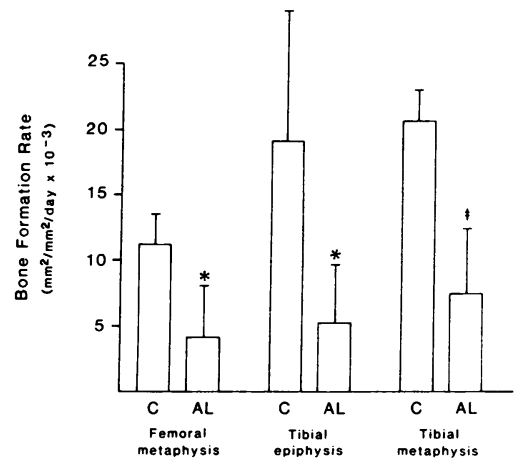

ble tetracycline labels, were not different in $\mathrm{C}$ and $\mathrm{Al}$ (Fig. 4). When expressed per unit length of active bone forming surface, the rate of mineralized bone formation was also similar in $\mathrm{C}$ and $\mathrm{Al}$ (Fig. 5). Thus, at sites of continued osteoblastic activity within the skeleton, the formation of mineralized bone at the cellular level was not impaired during the administration of aluminum.

The mean distance of the osteoid surface from the cement line was similar in $\mathrm{C}$ and $\mathrm{Al}$ at osteoid seams adjacent to double tetracycline labels (Table III). In contrast, the mean distance from the cement line to the osteoid surface was greater in $\mathrm{Al}$ than in $\mathrm{C}$ at osteoid seams that did not label with tetracycline (Table III). In control animals, osteoid surfaces adjacent to double tetracycline labels were located further from the cement line than osteoid surfaces that did not label with tetracycline. This difference was not observed in $\mathrm{Al}$ (Table III). Moreover, whereas the width of osteoid seams was similar at tetracycline-positive and at tetracycline-negative osteoid surfaces in C, osteoid seams were wider along unlabeled osteoid surfaces than at tetracyclinelabeled osteoid surfaces in $\mathrm{Al}$ (Table III).

\section{Discussion}

The results of the current investigation are consistent with previous reports in the $\operatorname{dog}(14)$ and in the rat $(12,13,15,27)$ and demonstrate that the administration of aluminum to experimental animals is associated with the development of osteomalacia in conjunction with evidence of aluminum deposition in bone. All aluminum-treated animals developed osteomalacia during the course of study as judged by established histologic criteria despite increases in the serum levels of both calcium and phosphorus and maintenance of normal renal function. The uptake of tetracycline into bone and the extent of osteoid seams covered with osteoblasts were each substantially reduced after $8 \mathrm{wk}$ of parenteral aluminum loading; these findings are qualitatively similar to results reported in clinical bone biopsy material from patients with aluminum-related osteomalacia $(1,4,6)$. The

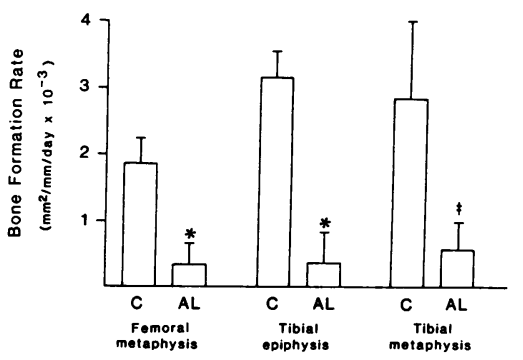

Figure 2. The rates of bone formation per unit length of osteoid surface in $\mathrm{C}$ and $\mathrm{Al}$ pigs at each of three skeletal sites. Values are the mean \pm 1 SD. ${ }^{*} P<0.01$ vs. $\mathrm{C} ; \ddagger P$ $<0.05$ vs. C.

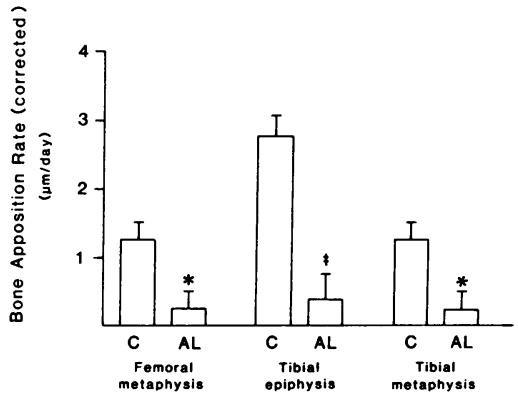

Figure 3. The corrected rates of mineralized bone apposition in $\mathrm{C}$ and $\mathrm{Al}$ pigs at each of three skeletal sites. The results represent the average width of mineralized bone deposited per day at osteoid surfaces during the time required to complete the calcification of an osteoid seam. Values are the mean \pm 1 SD. ${ }^{*} P<0.05$ vs. $\mathrm{C} ; \ddagger P<0.01$ vs. C.

aluminum content of bone in $\mathrm{Al}$ was also similar to values previously reported in such patients $(9,28)$. Consistent with observations in humans and in experimental animals with aluminumassociated osteomalacia, the rate of mineralized bone formation was markedly diminished in all aluminum-treated animals (15). Thus, both the histopathology of bone and several of the serum biochemical features in this experimental model of metabolic bone disease in the pig correspond to findings described in humans with aluminum-associated bone disease.

Reductions in the formation of mineralized bone are a prominent feature of aluminum-related bone disease (1-5). This change has been documented in both clinical and experimental studies (1-5, 10-14), and several groups of investigators have reported an inverse relationship between the aluminum content of bone and the rate of bone formation in patients with aluminum-associated bone disease $(2-4,28)$. The results of the current investigation are consistent with these earlier observations, but they also suggest that the disturbance in mineralized bone formation during aluminum administration is mediated by reductions in the number of sites of active bone formation, whereas the function of bone cells at these sites is not impaired.

In control animals, the rate of mineralized bone formation per unit area of tissue correlated with the percentage of trabecular bone surface that exhibited double tetracycline labels $(r=0.93$, $P<0.001)$. In contrast, no relationship was observed between the uncorrected rate of mineralized bone apposition and the rate of bone formation. Such findings indicate that the major determinant of mineralized bone formation at the tissue level in normal pigs is the number of active bone-forming sites, i.e., the total length of osteoid surface engaged in new bone formation, rather than variations in the specific cellular activity of osteoblasts at these sites. In aluminum-loaded animals, mineralized bone formation at the tissue level was substantially reduced from con-

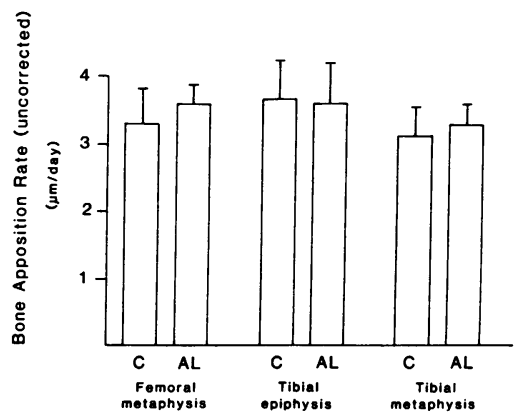

Figure 4. The uncorrected rates of mineralized bone apposition in $\mathrm{C}$ and $\mathrm{Al}$ pigs at each of three skeletal sites. The results represent the width of separation of double tetracycline labels divided by the time interval between the administration of the labels. Values are the mean $\pm 1 \mathrm{SD}$. 


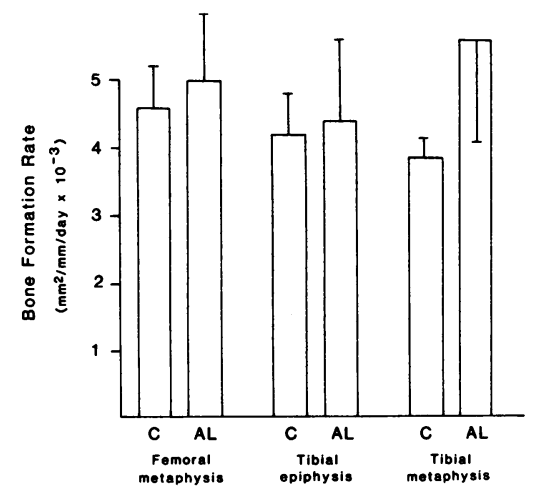

Figure 5. The rates of bone formation per unit length of active boneforming surface in $\mathrm{C}$ and $\mathrm{Al}$ pigs at each of three skeletal sites. Active bone forming surfaces were identified by the presence of double bands of tetracycline fluorescence adjacent to surface osteoid seams. Values are the mean \pm 1 SD.

trol values in conjunction with reductions in the extent of double tetracycline labeling, and fewer osteoid seams were characterized by the presence of osteoblasts in $\mathrm{Al}$. Thus, the number of sites of active bone formation was markedly altered by the administration of aluminum $(7,8,18)$. The corrected rates of mineralized bone apposition were also low in $\mathrm{Al}$, but the calculation of this variable of bone dynamics is based upon the fraction of osteoid surfaces that label with tetracycline $(7,8)$. Therefore, the corrected values for bone apposition only confirm the tissuebased measurements of bone formation in animals given aluminum.

In contrast to the findings at the tissue level, mineralized bone formation at the cellular level was not adversely affected by the administration of aluminum. The rate of bone formation, expressed per unit length of double tetracycline labeled bone surface, was similar in control and aluminum-treated animals. Both the width of separation of double tetracycline labels and, therefore, the uncorrected rates of mineralized bone apposition were similar in $\mathrm{C}$ and $\mathrm{Al}$. These results indicate that the function of osteoblasts was maintained at control levels along osteoid surfaces actively engaged in the formation of new mineralized bone in aluminum-treated animals $(7,8)$.

In the current study, reductions in the uptake of tetracycline into bone in $\mathrm{Al}$ could not be attributed to the presence of localized deposits of aluminum at the junction between mineralized bone and osteoid. An inverse relationship between the aluminum content of bone determined by histochemical methods and the extent of double tetracycline labeling was not observed; this was true whether the values for double tetracycline-

Table III. Estimates of Bone Matrix

(Osteoid) Production by Osteoblasts at the Epiphysis of the Proximal Tibia in C and Al Pigs ${ }^{* \neq}$

\begin{tabular}{|c|c|c|c|c|}
\hline & \multicolumn{2}{|c|}{$\begin{array}{l}\text { Tetracycline positive } \\
\text { osteoid surfaces }\end{array}$} & \multicolumn{2}{|c|}{$\begin{array}{l}\text { Tetracycline negative } \\
\text { osteoid surfaces }\end{array}$} \\
\hline & $\begin{array}{l}C \\
(n=4)\end{array}$ & $\begin{array}{l}\mathrm{Al} \\
(n=4)\end{array}$ & $\begin{array}{l}C \\
(n=4)\end{array}$ & $\begin{array}{l}\mathrm{Al} \\
(n=4)\end{array}$ \\
\hline $\begin{array}{l}\text { Distance of the oste } \\
\text { surface from the } \\
\text { cement line, } \mu \mathrm{m}\end{array}$ & $40.5 \pm 4.4$ & $49.6 \pm 8.8$ & $27.7 \pm 4.5^{8}$ & $42.1 \pm 12.0$ \\
\hline $\begin{array}{l}\text { Osteoid seam width, } \\
\boldsymbol{\mu \mathrm { m }}\end{array}$ & $5.3 \pm 0.5$ & $7.5 \pm 1.7^{11}$ & $5.8 \pm 0.9$ & $16.5 \pm 3.6^{\prime}$ \\
\hline
\end{tabular}

* Values are the mean \pm 1 SD.

* Probabilities determined by the $t$ test for unpaired samples.

$\checkmark P<0.01$ vs. tetracycline-positive $\mathrm{C}$.

" $P<0.05$ vs. tetracycline-positive C.

$' P<0.01$ vs. tetracycline-negative $C$. labeled surface were expressed as a percentage of the total bone surface or as a percentage of the osteoid surface. Also, in serial sections of bone obtained from each skeletal site and stained either for aluminum or examined by fluorescence microscopy, louble tetracycline labels were identified at osteoid seams that exhibited deposits of aluminum. The uptake of tetracycline into bone thus was not confined to osteoid seams at which aluminum had not deposited.

The current findings in experimental aluminum-induced osteomalacia are analogous to results previously reported using histomorphometric analyses in humans with early vitamin Ddeficient osteomalacia (8). In these studies, cellular indices of bone formation were normal despite substantial reductions in the rate of mineralized bone formation at the tissue level. As in the current experiment, the state of low bone formation in early vitamin D-deficient osteomalacia was also due primarily to reductions in the extent of active bone-forming surface (8). The relatively short duration of aluminum administration in the current study may account for the similarity between the dynamics of mineralized bone formation in aluminum-loaded pigs and those of early vitamin D-deficient osteomalacia in humans. Such observations suggest that reductions in the number of active bone-forming surfaces and in the total number of active osteoblasts represent important early changes in the evolution of both types of osteomalacia $(7,8)$. In support of this contention, Robertson et al. reported lower values for osteoblastic osteoid surface in rats with histologic osteomalacia induced by $12 \mathrm{wk}$ of daily intraperitoneal injections of aluminum (13). Reductions in the number of osteoblasts have also been described in bone biopsy material from patients with aluminum-associated osteomalacia $(3,5,6)$. Although aluminum may directly inhibit the mineralization of osteoid, the current experimental results thus suggest that the state of low bone formation in aluminum-induced osteomalacia is primarily due to fewer sites of active, cell-mediated osteoid formation and mineral deposition.

To further evaluate the cellular response of bone to aluminum administration, several indices of osteoblastic cell work were determined in each study group. For mineralized bone to form, an unmineralized collagenous bone matrix must first be deposited. The total volume of bone matrix, or osteoid, deposited at bone-forming surfaces is a function of the work of osteoblasts during periods of active collagen synthesis $(7,8,29)$. Therefore, measurements of the distance of the osteoid surface from the adjacent cement line represent the cumulative production of bone matrix by osteoblasts at existing osteoid seams. Based upon data obtained in humans with various types of metabolic bone disease, the distance of the osteoid surface from the cement line is diminished in conditions where the production of bone matrix by osteoblasts has terminated prematurely or under circumstances where the onset of calcification within newly formed osteoid seams has been substantially delayed $(7,8)$. Conversely, the distance of the osteoid surface from the cement line is normal if the progress of mineralized bone formation at existing boneforming surfaces has been slowed during the completion of an osteoid seam because of increased periods of cellular quiescence or temporary interruptions in mineralization $(7,8)$.

To distinguish among these possibilities, the distance of the osteoid surface from the cement line was determined both at tetracycline-positive and at tetracycline-negative osteoid surfaces in control and aluminum-treated animals. Double tetracycline labels were identified at various distances from the cement line in both groups of animals, and the mean distance of the osteoid 
surface from the cement line was not different in control and aluminum-treated animals at sites of double tetracycline labeling. These results suggest that the deposition of mineralized bone persisted throughout the course of osteoid synthesis at sites of continued bone formation in aluminum-treated animals. The modest increase from control values in osteoid seam width at double tetracycline-labeled osteoid surfaces in aluminum-loaded animals indicates that the rate of mineralized bone apposition was only slightly less than the rate of matrix apposition at sites of active mineralized bone formation.

In contrast, osteoid seams that did not show evidence of tetracycline uptake were identified at greater distances from the cement line in $\mathrm{Al}$ than in $\mathrm{C}$. Thus, the absence of tetracycline labeling along osteoid seams could not be attributed solely to delays in the initiation of calcification within newly formed osteoid seams or to the premature cessation of bone matrix synthesis at tetracycline-negative osteoid surfaces. Although not conclusive, the data are consistent with the interpretation that the accumulation of aluminum in bone leads to more prolonged periods of arrest of osteoblast function and to the temporary cessation of calcification along osteoid seams during the course of skeletal mineralization $(7,8,30)$. Such changes delay the completion of individual osteoid seams and lead an increase in the extent of osteoid surface in trabecular bone. Increases in the width of osteoid seams are particularly prominent at osteoid surfaces where mineralization has been interrupted (Table III).

The current data suggest that the total production of osteoid by osteoblasts within individual osteoid seams is not impaired during the administration of aluminum; however, the overall rate of deposition of new bone matrix by osteoblasts is almost certainly reduced. Considerable experimental evidence has been presented to indicate that matrix synthesis is impaired in aluminum-related bone disease $(10,11)$. Moreover, as shown in Fig. 3, the corrected rates of mineralized bone apposition in $\mathrm{Al}$ fall below those determined in $\mathrm{C}$ by a minimum of $1 \mu \mathrm{m} / \mathrm{d}$ at each skeletal site. Without concurrent and substantial reductions from control values in the linear rate of matrix deposition by osteoblasts in aluminum-loaded animals, the extent of osteoid accumulation and the width of osteoid seams would have considerably exceeded the values actually observed in aluminumtreated pigs.

Despite the similarity between the current experimental data and the skeletal findings in early vitamin D deficiency, it is unlikely that the histologic and dynamic response of bone to aluminum loading represents resistance to the actions of vitamin $\mathrm{D}$ at the tissue level. Hypocalcemia almost invariably occurs in vitamin $\mathrm{D}$ deficiency, and this disturbance is a major determinant of the defect in the calcification of osteoid in this disorder (3134 ). In contrast, the lesion of aluminum-related osteomalacia develops in the presence of normal or increased levels of calcium in serum (35). Quarles et al. noted that the accumulation of aluminum in bone did not prevent the resolution of vitamin $D$ deficient osteomalacia in dogs treated with vitamin $\mathrm{D}$ (36), and similar observations were reported by Hodsman et al. in the rat (37). However, the respective mineralization defects of vitamin D-deficient osteomalacia and aluminum-induced osteomalacia may not share a common mechanism. The reversal of a vitamin D-mediated disturbance in skeletal mineralization following treatment with vitamin $\mathrm{D}$ does not, therefore, exclude alterations in the calcification of osteoid that may occur by other mechanisms in association with aluminum accumulation in bone. This issue will require further study using in vitro techniques.
Both resorption surface and active formation surface decreased in aluminum-treated animals, and similar findings have been noted in bone biopsies from patients with aluminum-related bone disease $(3,5)$. It is possible that aluminum diminishes the formation of bone, and also its resorption, by affecting the differentiation of cellular precursors into osteoblasts and/or osteoclasts rather than by modifying the function of existing bone cells (38-40). Osteoclasts may be derived from precursor cells of bone marrow origin, most probably of the monocyte-macrophage series $(38,39)$. Changes in the differentiation of osteoclastic precursors into mature osteoclasts could account for reductions in the extent of resorption surface in aluminum-treated animals. Concurrent decreases in osteoblast numbers might occur secondarily by the mechanism of coupled bone resorption and bone formation (30) or directly by disturbances in the differentiation of mature osteoblasts $(7,40)$. This issue has yet to be evaluated experimentally, but alterations in the secretion of PTH (41-43), in the metabolism of vitamin D (14), or in the response of bone to either of these hormonal modulators of bone formation and resorption represent potential mechanisms by which both the number of osteoblasts and the number of osteoclasts may be reduced in aluminum-related bone disease.

In summary, the administration of aluminum reduces the number of sites of active bone formation in an experimental model of aluminum-related osteomalacia in the pig. The effect of aluminum on bone formation is mediated by decreases in the number of metabolically active osteoblasts and in the extent of active forming surface within bone. The mechanism responsible for such changes remains uncertain, but the function of active osteoblasts is not impaired during experimental aluminum loading. Whether similar reductions in the number of active osteoblasts are also present in the aplastic lesion of aluminumrelated bone disease remains to be established, but findings consistent with the current experimental results have been reported in bone biopsy material from such patients. The current data suggest that the accumulation of aluminum in bone may modify the function of osteoblasts in a manner distinct from a direct physical-chemical effect of aluminum to impair in the mineralization of osteoid.

\section{Acknowledgments}

The authors wish to thank Dr. R. L. Horst and the staff of his laboratory for providing the measurements of 1,25 dihydroxyvitamin $D$ in serum. Ms. Alina Fernandez, Ms. Jeanenne Gilligan, and Ms. Maria Bymakos provided excellent technical assistance, and Ms. Ellyn Boyd assisted in the preparation of the manuscript.

Dr. Sedman was supported, in part, by funds from Ross Laboratories, Columbus, $\mathrm{OH}$. Dr. Alfrey is a Medical Investigator of the Veterans Administration, and Dr. Goodman received support from the Research Service of the Veterans Administration.

\section{References}

1. Hodsman, A. B., D. J. Sherrard, E. G. C. Wong, A. S. Brickman, D. B. N. Lee, A. C. Alfrey, F. R. Singer, A. W. Norman, and J. W. Coburn. 1981. Vitamin D resistant osteomalacia in hemodialysis patients lacking secondary hyperparathyroidism. Ann. Intern. Med. 94:629-637.

2. Ott, S. M., N. A. Maloney, J. W. Coburn, A. C. Alfrey, and D. J. Sherrard. 1982. The prevalence of aluminum in renal osteodystrophy and its relationship to response to calcitriol therapy. N. Engl. J. Med. 307:709-713.

3. Llach, F., A. J. Felsenfeld, M. D. Coleman, and J. A. Pederson. 1984. Prevalence of various types of bone disease in dialysis patients. Proc. Ninth Int. Congr. Nephrol. 2:1375-1382.

4. Ott, S. M., N. A. Maloney, G. L. Klein, A. C. Alfrey, M. E. Ament, 
and J. W. Coburn. 1983. Aluminum is associated with low bone formation in patients receiving chronic parenteral nutrition. Ann. Intern. Med. 98:910-914.

5. Sherrard, D. J., S. M. Ott, N. A. Maloney, D. L. Andress, and J. W. Coburn. 1984. Uremic osteodystrophy: classification, cause and treatment. In Proceedings of the Symposium on Clinical Disorders of Bone and Mineral Metabolism. B. Frame and J. Potts, editors. Exerpta Medica, Amsterdam. 254-259.

6. Charhon, S. A., P. M. Chavassieau, M. C. Chapuy, G. Y. Boivin, and P. J. Meunier. 1985. Low rate of bone formation with or without histologic appearance of osteomalacia in patients with aluminum intoxification. J. Lab. Clin. Med. 106:123-131.

7. Parfitt, A. M. 1983. The physiologic and clinical significance of bone histomorphometric data. In Bone Histomorphometry: Techniques and Interpretation. R. R. Recker, editor. CRC Press, Boca Raton, FL. 143-223.

8. Parfitt, A. M., C. Mathews, D. Rao, B. Frame, M. Kleerekoper, and A. R. Villaneuva. 1981. Impaired osteoblast function in metabolic bone disease. In Osteoporosis: Recent Advances in Pathogenesis and Treatment. H. F. DeLuca, H. Frost, W. Jee, C. Johnson, and A. M. Parfitt, editors. University Park Press, Baltimore, MD. 321-330.

9. Maloney, N. A., S. M. Ott, A. C. Alfrey, N. L. Miller, J. W. Coburn, and D. J. Sherrard. 1982. Histological quantitation of aluminum in iliac bone from patients with renal failure. J. Lab. Clin. Med. 99:206-216.

10. Goodman, W. G., J. Gilligan, and R. Horst. 1984. Short-term aluminum administration in the rat: Effects on bone formation and relationship to renal osteomalacia. J. Clin. Invest. 73:171-181.

11. Goodman, W. G. 1984. Short-term aluminum administration in the rat: Reductions in bone formation without osteomalacia. J. Lab. Clin. Med. 103:749-757.

12. Chan, Y., A. C. Alfrey, S. Posen, D. Lissner, E. Hills, C. Dunstan, and R. Evans. 1983. Effect of aluminum on normal and uremic rats: Tissue distribution, vitamin $\mathrm{D}$ metabolites, and quantitative bone histology. Calcif. Tissue Int. 35:344-351.

13. Robertson, J., A. Felsenfeld, C. Haygood, and F. Llach. 1983. An animal model of aluminum $(\mathrm{AL})$ induced osteomalacia $(\mathrm{OM})$ : role of chronic renal failure (CRF) and parathyroid hormone (PTH). Kidney Int. 23:327-335.

14. Goodman, W. G., D. A. Henry, R. Horst, R. K. Nudelman, A. C. Alfrey, and J. W. Coburn. 1984. Parenteral aluminum administration in the dog. II. Induction of osteomalacia and effect on vitamin D metabolism. Kidney Int. 25:370-375.

15. Goodman, W. G. 1984. The differential response of cortical and trabecular bone to aluminum administration in the rat. Proc. Soc. Exp. Biol. Med. 179:509-516.

16. Sherrard, D. J., D. J. Baylink, J. E. Wergedal, and N. A. Maloney. 1974. Quantitative histological studies on the pathogenesis of uremic bone disease. J. Clin. Endocrinol. Metab. 39:119-135.

17. Villanueva, A. R., G. Czupinski, and A. M. Parfitt. 1979. A toluidine blue stain for calcification front, cement line, and osteoid seams on plastic embedded undecalcified sections of bone. J. Histotechnol. 2: 129.

18. Frost, H. M. 1983. Bone histomorphometry: analysis of trabecular bone dynamics. In Bone Histomorphometry: Techniques and Interpretation. R. R. Recker, editor. CRC Press, Boca Raton, FL. 109-131.

19. Lastgarten, J. A., and R. E. Wenk. 1972. Clin. Chem. 18:14191422 .

20. Fiske, C. H., and H. Y. Subbarow. 1925. The colorimetric determination of phosphorus. J. Biol. Chem. 66:375-400.

21. Heinegard, D., and G. Tidestrom. 1973. Determination of serum creatinine by a direct colorimetric method. Clin. Chem. Acta. 43:305311 .

22. Alfrey, A. C., G. R. LeGendre, and W. D. Kaehny. 1976. The dialysis encephalopathy syndrome: possible aluminum intoxication. $N$. Engl. J. Med. 294:184-188.

23. Horst, R. L., E. T. Littledike, J. L. Riley, and J. L. Napoli. 1981. Quantitation of vitamin D and its metabolites and their plasma concentrations in five species of animals. Ann. Biochem. 116:189-203.
24. Reinhardt, T. A., R. L. Horst, J. W. Orf, and B. W. Hollis. 1984. A microassay for 1,25 dihydroxyvitamin $D$ not requiring high performance liquid chromatography. J. Clin. Endocrinol. Metab. 58:91-98.

25. Horst, R. L., and E. T. Littledike. 1982. Comparison of plasma concentrations of vitamin $\mathrm{D}$ and its metabolites in growing and aged domestic animals. Comp. Biochem. Biophys. 73b:485-489.

26. Dixon, W. J., and F. J. Massey, editors. 1969. Introduction to statistical analysis. McGraw-Hill Book Co., New York. 75-94, 150192, 335-345.

27. Ellis, H. A., J. H. McCarthy, and J. Herrington. 1979. Bone aluminum in hemodialysed patients and in rats injected with aluminum chloride: relationship to impaired bone mineralization. J. Clin. Pathol. 32:832-844.

28. Hodsman, A. B., D. J. Sherrard, A. C. Alfrey, S. Ott, A. S. Brickman, N. L. Miller, N. A. Maloney, and J. W. Coburn. 1982. Bone aluminum and histomophometric features of renal osteodystrophy. J. Clin. Endocrinol. Metab. 54:539-546.

29. Parfitt, A. M. 1976. The actions of parathyroid hormone on bone: relation to bone remodeling and turnover, calcium homeostasis, and metabolic bone disease. I. Mechanisms of calcium transfer between blood and bone and their cellular basis: morphologic and kinetic approaches to bone turnover. Metab. Clin. Exp. 25:809-844.

30. Parfitt, A. M. 1976. The actions of parathyroid hormone on bone: Relation to bone remodeling and turnover, calcium homeostasis, and metabolic bone disease. III. PTH and osteoblasts, the relationship between bone turnover and bone loss, and the state of the bone in primary hyperparathyroidism. Metab. Clin. Exp. 25:1033-1068.

31. Baylink, D. J., M. Stauffer, J. Wergedal, and C. Rich. 1970. Formation, mineralization, and resorption of bone in vitamin $\mathrm{D}$ deficient rats. J. Clin. Invest. 49:1122-1134.

32. Frame, B., and A. M. Parfitt. 1978. Osteomalacia: current concepts. Ann. Intern. Med. 89:966-982.

33. Stauffer, M., D. Baylink, J. Wergedal, and C. Rich. 1973. Decreased bone formation, mineralization, and enhanced resorption in calcium-deficient rats. Am. J. Physiol. 225:269-276.

34. Howard, G. A., and D. J. Baylink. 1980. Matrix formation and osteoid maturation in vitamin D-deficient rats made normocalcemic by dietary means. Miner. Electrolyte Metab. 3:44-50.

35. Coburn, J. W. 1984. Role of aluminum accumulation in renal osteodystrophy. Proc. Ninth Int. Congr. Nephrol. 2:1383-1395.

36. Quarles, L. D., V. W. Dennis, H. J. Gitelman, J. M. Harrelson, and M. K. Drezner. Aluminum deposition at the osteoid-bone interface. An epiphenomenon of the osteomalacic state in vitamin D-deficient dogs. J. Clin. Invest. 75:1441-1447.

37. Hodsman, A. B., B. Steer, and M. Bishop-Michniewicz. 1983. Vitamin D can reverse osteomalacia in aluminum loaded vitamin Ddeficient rats. In Proceedings of the Fifth Annual Scientific Meeting, American Society for Bone and Mineral Research, San Antonio, TX. A35 (Abstr).

38. Mundy, G. R. 1983. Monocyte-macrophage system and bone resorption. Lab. Invest. 49:119-121.

39. Kahn, A. J., C. C. Stewart, and S. L. Teitelbaum. 1978. Contactmediated bone resorption by human monocytes in vitro. Science (Wash. DC). 199:988-990.

40. Parfitt, A. M. 1976. The actions of parathyroid hormone on bone: relation to bone remodeling and turnover, calcium homeostasis, and metabolic bone disease. II. PTH and bone cells: bone turnover and plasma calcium regulation. Metab. Clin. Exp. 25:909-955.

41. Kraut, J. A., J. H. Shinaberger, F. R. Singer, D. J. Sherrard, J. Saxton, J. H. Miller, K. Kurokawa, and J. W. Coburn. 1983. Parathyroid gland responsiveness to acute hypocalcemia in dialysis osteomalacia. Kidney Int. 23:725-730.

42. Andress, D. L., A. Voights, A. Felsenfeld, and F. Llach. 1983. Parathyroid hormone response to hypocalcemia in dialysis patients with osteomalacia and osteitis fibrosa. Kidney Int. 24:364-369.

43. Morrissey, J., M. Rothstein, G. Mayor, and E. Slatopolsky. 1983. Suppression of parathyroid hormone secretion by aluminum. Kidney Int. 23:699-704. 